\title{
Comparison of Huelsmann Basic Biquad with View to the Decrease of the Attenuation over the Transient Frequency of the Operational Amplifier
}

\author{
BOHUMIL BRTNÍK \\ DAVID MATOUŠEK \\ MIROSLAV STEHLÍK \\ VOJTĚCH STEJSKAL \\ Department of Electrical Engineering \\ University of Pardubice \\ Náměstí Čs. Legií, 53002 Pardubice \\ CZECH REPUBLIC \\ bohumil.brtnik@upce.cz, david.matousek@upce.cz,
}

\begin{abstract}
The signal processing at discrete time requires an aliasing filter at the input and a reconstruction filter at its output. In this paper, selected basic structures of biquads are described and compared with a view to the decrease of the attenuation over the transient frequency of the operational amplifier. Concretely, low-pass and band-pass Huelsman (i.e. multi feedback) structure are confronted with the limits of a real operational amplifier. Firstly, the reasons of the decreasing of the attenuation are explained theoretically. Secondly, these conclusions are verified by simulations. These simulations were performed by spice-like circuit simulator MicroCap version 10.
\end{abstract}

Key Words: Low-pass, Band-pass Filters Structure, Real Operational Amplifier; Frequency Response, Decreasing of the Attenuation at High Frequencies.

Received: March 18, 2020. Revised: September 2, 2020. Accepted: November 4, 2020. Published: November 23, 2020.

\section{Introduction}

Circuits that process the signal at a discrete time, i.e. switched capacitor circuits, switched currents and digital signal processing circuits, [1] require for their operation an aliasing filter at the input and a reconstruction filter at its output. These filters should have low pass filter and/or (in special causes) band-pass properties, but the use in active RC (ARC) filters of the operational amplifiers with real properties will modify the monotone-decreasing frequency response.

Some biquad filters structures are characterized by a decreasing of the attenuation at high frequencies [2], [3]. This decreasing of the attenuation occurs only for the filters of the even orders, i.e. for the biquads.

The cause of these attenuation losses is depicted in Fig. 1 for a low-pass filter of the second order [4], [5]. The ideal case of the frequency response of the low-pass filter of the second-order is the decreasing of the magnitude over cut-off frequency $\omega_{0}$ with $-40 \mathrm{~dB} / \mathrm{dec}$ slope. The finite value of the transient frequency $\omega_{\text {T }}$ of the used operational amplifier leads to the break of this ideal slope. The result is a loss of attenuation in the stopband for the frequencies around the transient frequency $\omega_{\mathrm{T}}$.

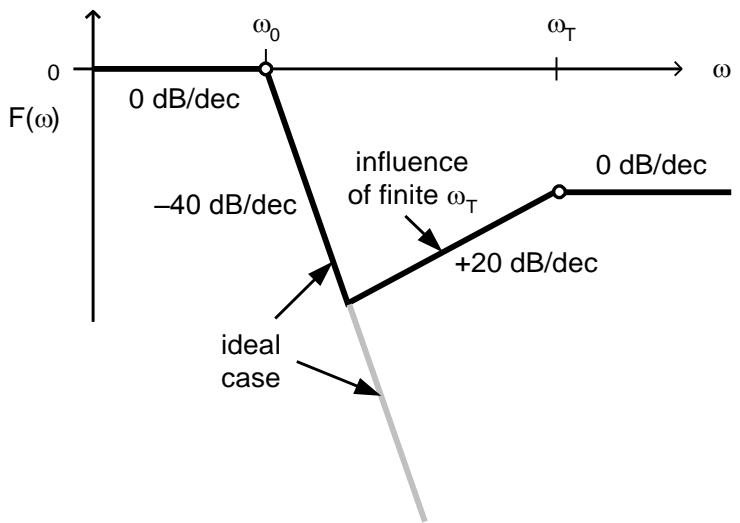

Figure 1. Fig.1 Frequency response of the low-pass biquad.

Filter with a distributed feedback loop using only one operational amplifier in Huelsmann general structure is depicted in Fig. 2 [6], [7]. Its transfer function $\mathrm{F}$ simply derived from this circuit diagram 
by signal flow graph (SFG) method (when the Mason formula is applied) is (1).

Figure 2.

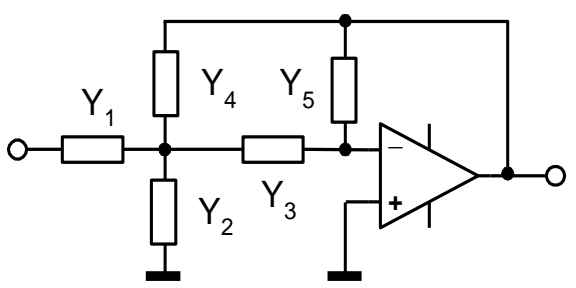

Fig.2 General Huelsmann filter structure.

$$
\mathrm{F}=\frac{-\mathrm{Y}_{1} \cdot \mathrm{Y}_{3}}{\left(\mathrm{Y}_{1}+\mathrm{Y}_{2}+\mathrm{Y}_{3}+\mathrm{Y}_{4}\right) \cdot \mathrm{Y}_{5}+\mathrm{Y}_{3} \cdot \mathrm{Y}_{4}}
$$

where $\mathrm{Y}$ are admittances, i.e. there are realized from $\mathrm{R}$ and/or $\mathrm{C}$ members.

\section{Problem Formulation}

Next turn our attention into some Huelsman filter structure, where will be analyzed and decreasing of the attenuation at high frequencies will be discussed.

\subsection{Low-pass Huelsmann filter structure}

Generally, the members of low-pass filter structure are derived from equation (2) [8], [9], [10], [11], where on the left side is the Huelsmann filter general transfer function, on the right side low-pass the general transfer function.

$$
\begin{gathered}
\frac{-Y_{1} \cdot Y_{3}}{Y_{1} \cdot Y_{5}+Y_{2} \cdot Y_{5}+Y_{3} \cdot Y_{5}+Y_{4} \cdot Y_{5}+Y_{3} \cdot Y_{4}}= \\
=\frac{\omega_{0}^{2}}{s^{2}+s \cdot \frac{\omega_{0}}{Q}+\omega_{0}^{2}}
\end{gathered}
$$

where: $\mathrm{Q}$ is quality, $\omega_{0}$ the corner frequency. Equation (2) nominator on the left side must be the real number because the nominator on the right side is the real number as well, therefore the admittances must be realized by resistors with the conductances as follows: ${ }^{Y_{1}}=G_{1}, Y_{3}=G_{3}$. Other admittances in the denominator can be choose as capacitors: $\mathrm{Y}_{4}=\mathrm{sC}_{4}, \mathrm{Y}_{5}=\mathrm{sC}_{5}$ and other as an resistor with the conductance $\mathrm{Y}_{2}=\mathrm{G}_{2}$. In this case
(LP-H version 1), corresponding circuit diagram is depicted in Fig. 3.

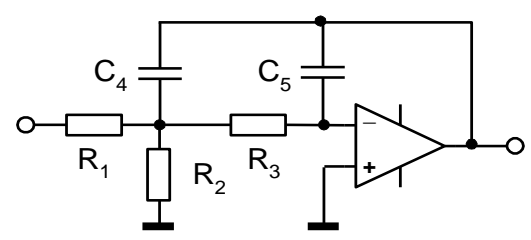

Fig.3 LP-H filter structure ver. 1.

Next, the circuit magnitude characteristics of this circuit diagram were analyzed by simulation, the program MicroCap version 10 is used, and the result is given in Fig. 4.

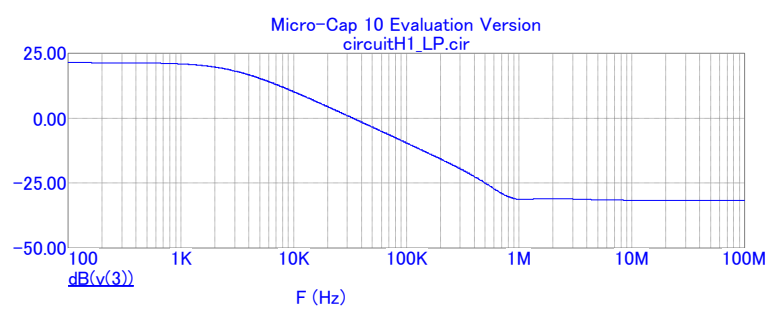

Fig.4 LP-H filter structure ver. 1 frequency response.

The output voltage of the frequency-independent divider: $R_{1}, R_{2}$ is exciting other parts of this circuit, thus output voltage LP-H filter ver. 1 is not zero at a higher frequency. As we can see, in this case, the structure has not unfortunately and monotone increasing attenuation in the stopband. The lowest attenuation the magnitude $30 \mathrm{~dB}$ only over the transient frequency $\omega_{\mathrm{T}}$ seems very small, it is not so quite.

Consider now second one configuration (i.e. version 2), where nominator must be realized from resistors: $Y_{1}=G_{1}, Y_{3}=G_{3}$ as well, but in the denominator, we can choose as admittances: $\mathrm{Y}_{2}=\mathrm{sC}_{2}, \quad \mathrm{Y}_{5}=\mathrm{sC}_{5}$ as capacitors and $\mathrm{Y}_{4}=\mathrm{G}_{4}$ as the resistor. Thus, the corresponding circuit diagram is depicted in Fig. 5.

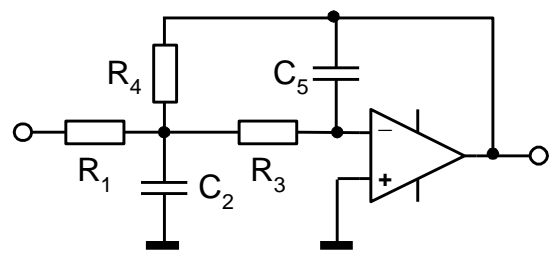

Fig.5 LP-H filter structure ver. 2.

The magnitude characteristic of this circuit diagram will be now analyzed with simulation by the 
program MicroCap version 10, and the result is given in Fig. 4.

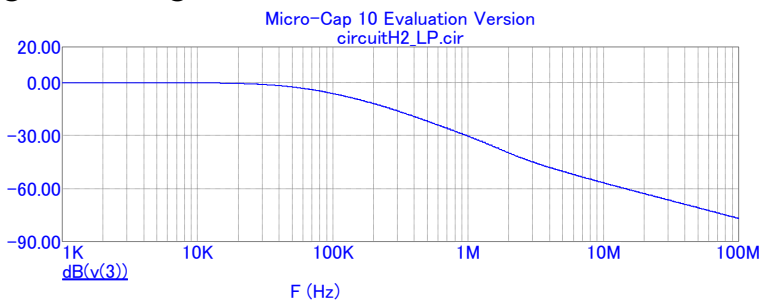

Fig.6 LP-H filter structure ver. 2 frequency response.

The members: $\mathrm{R}_{1}, \mathrm{C}_{2}$ are frequency-dependent voltage divider, at highest frequency is capacitor: $\mathrm{C}_{1}$ as short circuit, in this case: $\mathrm{V}_{\mathrm{C} 2}=0$. Thus, its output voltage (i.e. $\mathrm{V}_{\mathrm{C} 2}$ ) has a monotone increasing. As we can see from simulation, the biquad in this structure version 2 has a monotone increasing attenuation in stopband. Therefore, this version 2 is recommended for application.

\subsection{Band-pass Huelsmann filter structure}

In some cases, it is necessary to use the band-pass filter as well. Generally, the members of low-pass filter structure are derived from equation (3), where on the left side is the Huelsmann filter general transfer function, on right side the band-pass general transfer function.

$$
\begin{gathered}
\frac{-Y_{1} \cdot Y_{3}}{Y_{1} \cdot Y_{5}+Y_{2} \cdot Y_{5}+Y_{3} \cdot Y_{5}+Y_{4} \cdot Y_{5}+Y_{3} \cdot Y_{4}}= \\
=\frac{s \cdot \frac{\omega_{0}}{Q}}{s^{2}+s \cdot \frac{\omega_{0}}{Q}+\omega_{0}^{2}}
\end{gathered}
$$

Equation (3) nominator must be a complex number in this case, therefore the admittances must be realized by resistors as follows: ${ }^{Y_{1}}=\mathrm{G}_{1}$. Another admittance can be chosen as a capacitor: $\mathrm{Y}_{4}=\mathrm{sC}_{4}$ and $\mathrm{Y}_{2}=\mathrm{G}_{2}, \mathrm{Y}_{5}=\mathrm{G}_{5}$ must be realized as the resistors. The corresponding circuit diagram, in this case, is depicted in Fig. 7.

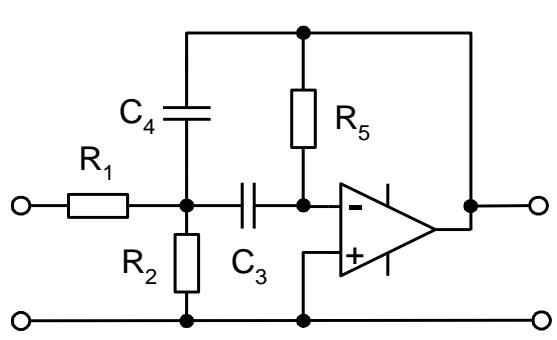

Fig.7 BP-H filter structure ver. 1.

The output voltage of frequency-independent input divider: $R_{1}, R_{2}$ is exciting other parts of this circuit, thus output voltage BP-H filter ver. 1 is not zero at a higher frequency. Next, this circuit diagram will be analyzed with simulation by program MicroCap version 10, the result is given in Fig. 8.

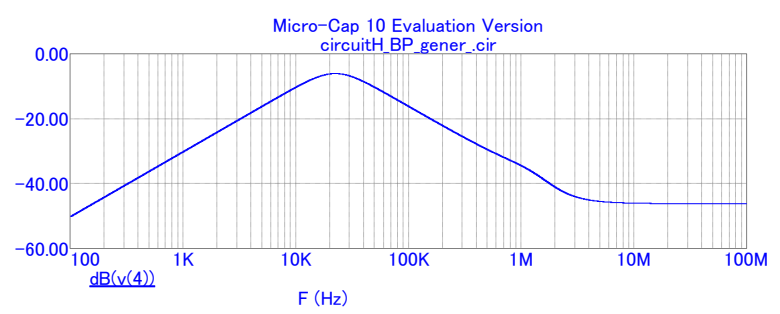

Fig.8 Filter BP-H structure ver. 1 frequency response.

As we can see, this biquad structure has not unfortunately a monotone increasing attenuation in stopband.

Consider now second one configuration (i.e. ver. 2), where nominator must be realized as: $\mathrm{Y}_{1}=\mathrm{G}_{1}, \mathrm{Y}_{3}=\mathrm{sC}_{3}$ as well, but in the denominator we can choose admittances: $\mathrm{Y}_{2}=\mathrm{sC}_{2}$, $\mathrm{Y}_{5}=\mathrm{sC}_{5}$ realized as capacitors and $\mathrm{Y}_{4}=\mathrm{G}_{4}$ as the resistor. Thus, the corresponding circuit diagram is depicted in following Fig. 9.

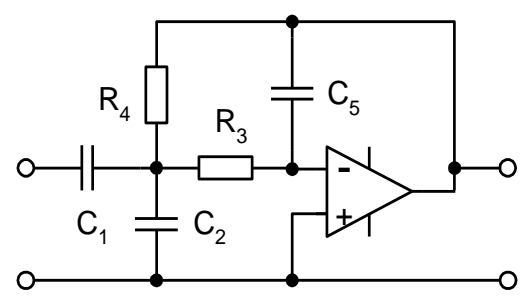

Fig.9 BP-H filter inversion structure (ver. 2).

Notice: As we can see in the comparison between Fig. 7 (ver. 1) and Fig. 9 (ver. 2), all passive 
elements are inverted, because resistors and capacitors are changed.

The capacitors: $\mathrm{C}_{1}$ and $\mathrm{C}_{2}$, connected into the same node, are no short circuits in this case (in comparison with Fig. 5), because they are the input voltage divider. This divider is frequency independent, as is depicted in equation (4).

$$
\frac{V_{\mathrm{C} 2}}{V_{\mathrm{D}}}=\frac{\frac{1}{s \mathrm{C}_{2}}}{\frac{1}{s \mathrm{C}_{1}}+\frac{1}{s \mathrm{C}_{2}}}=\frac{\frac{1}{s \mathrm{C}_{2}}}{\frac{s \mathrm{C}_{1}+s \mathrm{C}_{2}}{\mathrm{sC}_{1} \cdot s \mathrm{C}_{2}}}=\frac{\mathrm{C}_{1}}{\mathrm{C}_{1}+\mathrm{C}_{2}}
$$

where: $s=j \omega$. Thus output voltage of divider: $C_{1}$, $\mathrm{C}_{2}$ (i.e. $\mathrm{V}_{\mathrm{C} 2}$ ) is exciting other parts of this circuit in all frequencies. In this reason, output voltage BP-H filter ver. 2 is therefore not zero at higher frequencies as well.

This circuit diagram will in next step analyzed by simulation with program MicroCap version 10, the result is given in Fig. 10. As we can see, these conclusions are verified by simulations very well, it is depicted, that this biquad structure has not a monotone increasing attenuation in stop band unfortunately as well.

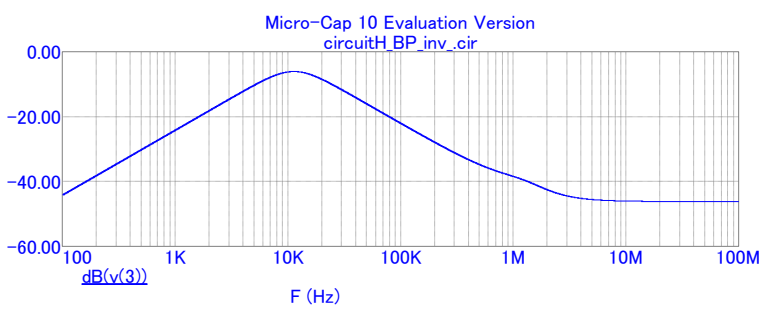

Fig.10 BP-H filter inversion structure frequency response (ver. 2).

For all simulations were the following value used: $\mathrm{R}=1.10^{3} \mathrm{Ohm}$ for resistors and $\mathrm{C}=1 \cdot 10^{-9} \mathrm{~F}$ for capacitors, operational amplifier LM741 with $\mathrm{R}_{\text {outac }}=50 \mathrm{Ohm}$, GBW $=1 \mathrm{MHz}$.

\section{Conclusion}

All low-pass and band-pass Huelsman (i.e. multi feedback) structure are discussed. Since the main function of both the low-pass (and/or by-pass filter in especial cases) is to suppress the higher frequency, the Huelsmann low-pass structure ver. 1 and both the band-pass filter structure cannot be recommended as an ideal filter. Only low-pass structure ver. 2 has a monotone increasing attenuation in all stopband (i.e. after the transient frequency of real operational amplifier as well), therefore only in this structure can be an ideal LP-H filter realised, as shows the results of the computer simulations. If the Huelsmann LP and/or BP structure filter is designed, the described reasons must be considered.

When the Huelsmann LP and/or BP structure filter is designed, we choose the same capacitors in the first step, the resistors are calculated from design equations next. The result from theoretical discussion and simulation is: it seems can be useful to find the new design process when will be $\mathrm{C}_{2}>\mathrm{C}_{1}$ for BP-H ver. 2. Consider for example

BP-H ver. $2, C_{2}=10 \cdot C_{1}, R_{4}=R_{3}$ where there is a frequency shift unfortunately, the result of simulation, in this case, is depicted in Fig. 11.

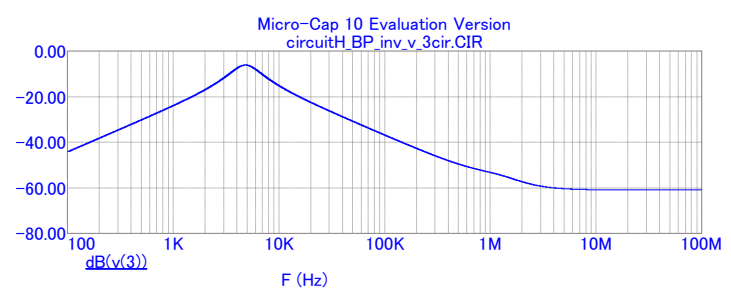

Fig.11 BP-H filter inversion structure frequency response (ver. 2), $R_{4}=R_{3}, C_{2}=10 \cdot C_{1}$.

Thus, the attenuation is increasing from $46 \mathrm{~dB}$ to $61 \mathrm{~dB}$ as we can see. In this case transfer $\frac{\mathrm{V}_{\mathrm{C2}}}{\mathrm{V}_{\mathrm{DN}}}$ of the operational amplifier transient frequency $\omega_{\text {T }}$ will be not zero, but lowest. This can be in some cases quite sufficient.

\section{Acknowledgement}

This work was supported by internal grant University of Pardubice, Department of Electrical Engineering.

References:

[1] F. Yuan, A. Opal, Computer Methods for Analysis of Mixed-Mode Switching Circuits, Kluever Academic Publisher, New York, 2004.

[2] L. Thede, Practical Analog and Digital Filter Design, Artech House, 2004.

[3] R. Mancini, Op Amps For Everyone - Design Reference, Texas Instruments, 2002

[4] J. Puncochar, Low Pass Filters Sallen and Key With Real Operational Amplifiers, Elektrorevue 10, 2005, pp. 1-13. 
[5] J. Dostal, Operational Amplifiers. BEN publisher, Prague, 2006.

[6] J. Bicak, M. Leipert, M. Vlcek, A Linear Circuits an Systems, CTU publisher, Prague, 2007.

[7] T. Dostal, K. Vrba, The Electric Filters, PC-DIR, Brno, 1997.

[8] D. Biolek, Solving Electronic Circuits. BEN publisher, Prague, 2004.

[9] W. Jung, Op Amp Applications Handbook, Elsevier, Oxford, UK, 2005, pp.307-419.

[10] K. Hájek, J. Sedláček, Frequency Filters, Ben publisher, Prague, 2006.

[11] P. Martinek, P. Boreš, J. Hospodka, Electrics Filters, CTU publisher, Praque, 2003.

\section{Creative Commons Attribution License 4.0} (Attribution 4.0 International, CC BY 4.0)

This article is published under the terms of the Creative Commons Attribution License 4.0

https://creativecommons.org/licenses/by/4.0/deed.en US 